\title{
Enteric Hepatitis: A case report
}

Nepal A', Acharya R', Ojha AR' ${ }^{2}$, Sah $\mathbf{S}^{3}$

${ }^{1}$ Residents, ${ }^{2}$ Assistant Professor, ${ }^{3}$ Medical Officer

Department of Paediatrics, Kathmandu Medical College Teaching Hospital, Kathmandu, Nepal

\section{Abstract}

Enteric fever is a common cause of morbidity and hospital admission in developing countries. This can sometimes be complicated by rare complications like hepatitis. One should be careful when selecting antibiotics like ceftriaxone in such children which can cause biliary stasis. Our case of enteric hepatitis treated with ceftriaxone developed a prolonged jaundice with biliary stasis as a result of antibiotic therapy.

Key words: Ceftriaxone, Enteric fever, Icterus

\section{INTRODUCTION}

$T^{\prime}$ yphoid fever is a common bacterial infection in the tropics attended with considerable morbidity and mortality ${ }^{1}$. Kathmandu, the capital city of Nepal, has been previously coined as enteric fever capital of the world. The population of Kathmandu is increasing and available figures suggest that enteric fever caused by Salmonella serovars Typhi and Paratyphi A show no signs of reduction in prevalence 1 . Nepal falls in the geographic zone having highest incidence of typhoid fever i.e. $>100$ per 100,000 per year ${ }^{2}$. Typhoid fever is caused by Salmonella enterica serovar Typhi (S.Typhi), a gramnegative bacterium. It occurs through ingestion of the organism via variety of sources of faecal contamination including street foods and contamination of water reservoirs ${ }^{3}$.

In tropics, typhoid is invariably kept as a differential diagnosis in any fever ${ }^{1}$. It commonly presents with acute enteritis, followed by bacteraemia and extra intestinal focal infection. It involves almost all major systems in the body due to bacteraemia and liver involvement may also occur as a part of it. It is associated with abnormal liver biochemical tests, but severe hepatic involvement with a clinical feature of acute hepatitis is a rare complication ${ }^{4}$.

Here we are discussing a child with enteric fever with a rare complication of hepatitis.

Address for correspondence

Dr. Archana Nepal

First year MD resident, Department of Paediatrics

Kathmandu Medical College Teaching Hospital, Sinamangal

Kathmandu, Nepal

E-mail: archuuana@gmail.com

\section{CASE REPORT}

A thirteen year old male child presented with history of high grade fever and abdominal pain for five days associated with vomiting, constipation, and passage of dark yellow coloured urine and yellowish discolouration of sclera. On examination, he was febrile and toxic. His pulse rate was disproportionately low for his temperature i.e. pulse of 64 beats per minute with temperature of $101^{\circ} \mathrm{F}$. He was icteric and had hepatomegaly (liver palpable three $\mathrm{cm}$ below subcostal margin in right mid-clavicular line), splenomegaly (spleen palpable $1.5 \mathrm{~cm}$ below left subcostal margin). His laboratory parameters revealed total count $5,400 / \mathrm{mm}^{3}$; serum glutamic oxaloacetic transaminase (SGOT): $4312 \mathrm{U} / \mathrm{L}$, serum glutamic pyruvic transaminase (SGPT): 3949 U/L, Alkaline phosphatase (ALP): $860 \mathrm{U} / \mathrm{L}$; Total serum bilirubin (TSB): $5.2 \mathrm{mg} / \mathrm{dl}$, direct serum bilirubin (DSB): $3.9 \mathrm{mg} / \mathrm{dl}$, Prothrombin time (PT): 22 seconds (control: 13 seconds). Widal agglutination test was positive for $\mathrm{O}$ and $\mathrm{H}$ antigen in 320 dilution and same test done prior to hospital admission was negative. Ultrasonography (USG) of abdomen showed hepatomegaly and splenomegaly. Blood culture for Salmonella was negative.

He was admitted with the provisional diagnosis of enteric hepatitis and was started on intravenous Ceftriaxone. Patient improved clinically on successive days but yellowish discoloration of sclera became deeper and he also complained of pruritis. Repeat liver function tests (LFT) after five days showed better liver function except for elevated Total serum bilirubin/Direct serum bilirubin. USG abdomen showed hepatomegaly with gall bladder sludge. Patient was continued on intravenous 
ceftriaxone for seven days and was discharged with oral Cefixime for seven more days.

LFT was repeated after one week of completion of total antibiotic therapy which showed reduction in liver enzymes, ALP, TSB, DSB.

\section{DISCUSSION}

Enteric fever is a common disease in our part of the world and diagnosing it in children is challenging. The gold standard for diagnosing typhoid fever is blood culture and blood culture positivity varies with different laboratories. The recent unpublished data of Kathmandu Medical College Teaching Hospital of three months (2069) for Salmonella blood culture is four percent. Many of the children will be already on antibiotics before they present to the hospital. Our case was also already on oral antibiotic at the time of presentation. So, the diagnosis of enteric fever was based on clinical parameters and positive widal test ${ }^{5}$.

Enteric fever is a disease with variety of complications due to involvement of different organ systems; Central Nervous System three to 35\%; Hepato-biliary one to 26\%; pulmonary one to six percent; Cardiovascular System one to five percent ${ }^{3}$. Since the earliest description of hepatic involvement in typhoid by Osler ${ }^{4}$, who documented enlarged tender liver with clinical jaundice in eight out of 1500 cases of typhoid fever, a number of studies have appeared showing isolated hepatic enlargement with or without splenomegaly in 13 to $65 \%$ of cases $^{4}$. Abnormal biochemical tests suggestive of hepatic involvement without enlargement of liver have been reported in 23$60 \%$ cases $^{4}$.

It has been suggested that mild increase in transaminase levels occurs in typhoid hepatitis which differentiate such cases from viral hepatitis ${ }^{4}$. However our case had a marked increase in liver enzymes along with increase in PT and International Normalised Ratio (INR). For exclusion of hepatitis as a cause for these results we sent serology for hepatitis A, E which was non-reactive. These two hepato-trophic viruses are the most common cause of hepatitis in children; however there are other viruses which can cause hepatitis which has not been ruled out in our case. Nevertheless, S.N Khosla also observed high transaminase level in all the cases of typhoid hepatitis included in his study ${ }^{4}$.

Liver involvement in enteric hepatitis may be in the form of hepatomegaly alone, jaundice, bio-chemical alterations and histopathological changes ${ }^{4}$. The possible associated factors for development of salmonella hepatitis are virulence of the organisms, delayed treatment and poor general health of the patients. The pathogenesis of severe hepatic involvement in salmonella infection may be multifactorial and includes endotoxin, local inflammatory and/or host immune reactions ${ }^{3}$.

Clinical jaundice in salmonella hepatitis usually occurs within the first two weeks of the febrile illness. The prognosis is usually good if there is early institution of therapy as salmonella hepatitis responds well to a specific antibiotic and jaundice resolves with clinical improvement ${ }^{4}$. Our patient showed clinical improvement with treatment however, resolution of jaundice did not occur. This could be due to the fact that Ceftriaxone was used for the treatment of enteric fever. Ceftriaxone is a third generation cephalosporin with long half-life, wide spectrum, high tissue penetration and good safety profile ${ }^{6}$. Liver involvement in the form of hepatitis has been reported with Ceftriaxone as a part of idiosyncratic drug reaction, where there will be elevation in the biochemical markers (ALT, AST). However, in our case repeat LFT showed decrease in level of liver enzymes and elevation in the level of total and direct bilirubin. This can be explained by the fact that Ceftriaxone calcium salt gets precipitated in the gall bladder causing biliary sludge and decreased excretion of bilirubin via bile, hence causing increased level of bilirubin in blood. Again Ceftriaxone binds with albumin and causes displacement of bilirubin causing its increased level in blood ${ }^{7}, 8$.

Liver function test repeated after 7 days of completion of therapy showed normalization of the hepatic enzymes and reduction of bilirubin level.

\section{CONCLUSION}

Enteric hepatitis though not a common entity should be recognized in any case of fever who develops jaundice at peak of fever. A correct diagnosis and early institution of therapy improves prognosis in these cases. 


\section{REFERENCES}

1. Karkey A, Aryjal A, Basnyat B, Baker B. Kathmandu, Nepal: Still an enteric fever capital of the world. J Infect Developing Countries. 2008;2(6):461-5.

2. Crump JA, Luby SP, Mintz E. The global burden of typhoid fever. Bull World Health Organ. 2004;82:346-53.

3. Bhutta ZA, Elder JS, Kleigmen RM, Schor NF, Geme WST. Nelson Text book of Pediatrics. 19th ed. India; Elsevier: 2011.

4. Khosla SN. Typhoid fever. Postgrad Med J. Nov 1990;66(781):923-5.

5. Choo KE, Razif AR, Oppenheimer SJ, Ariffin WA, Lau J, Abraham T. Usefulness of the Widal test in diagnosing childhood typhoid fever in endemic areas. J Paediatr Child Health. Feb 1993;29(1):36-9.

6. Peker E, Cagan E, Dogan M. Ceftriaxone-induced toxic hepatitis. World J Gastroenterol. June 2009;15(21):2669-71.

7. Ceftriaxone as the disodium salt [Internet]. [place of unknown]: 2011 Dec 1. Available from: http://www.medsafe.govt.nz/profs/datasheet/c/ ceftriaxoneaftinj.pdf

8. Gulian JM, Dalmasso C, Pontier F, Gonard V. Displacement effect of ceftriaxone on bilirubin bound to human serum albumin. Chemotherapy. 1986;32(5):399-403. 